Supporting Information: Luminescent ruthenium(II)- and rhenium(I)-diimine wires bind nitric oxide synthase.

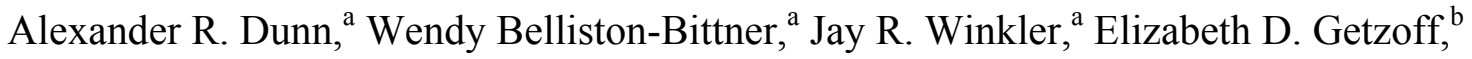
Dennis J. Stuehr, ${ }^{\mathrm{c}}$ Harry B. Gray ${ }^{\mathrm{a} *}$

a) Beckman Institute, California Institute of Technology, Pasadena, California 91125, b) Department of Molecular Biology and Skaggs Institute for Chemical Biology, The Scripps Research Institute, MS MB4, 10550 N. Torrey Pines Rd., La Jolla, CA 92037, c) Department of Immunology, Lerner Research Institute, The Cleveland Clinic, Cleveland, Ohio 44195.

\title{
Supplemental Spectra:
}

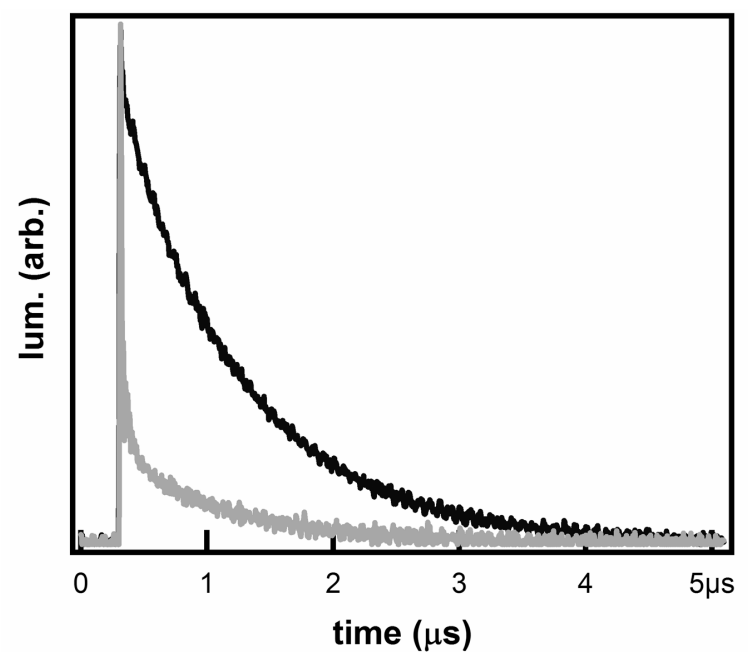

Supplemental Figure 1: Sample transient luminescence decay data for 5 (black) and a

1:1 mixture of 5 and $\Delta 114(9 \mu \mathrm{M}$; gray). The fast component of the luminescence decay corresponds to 5 bound to $\Delta 114$. 


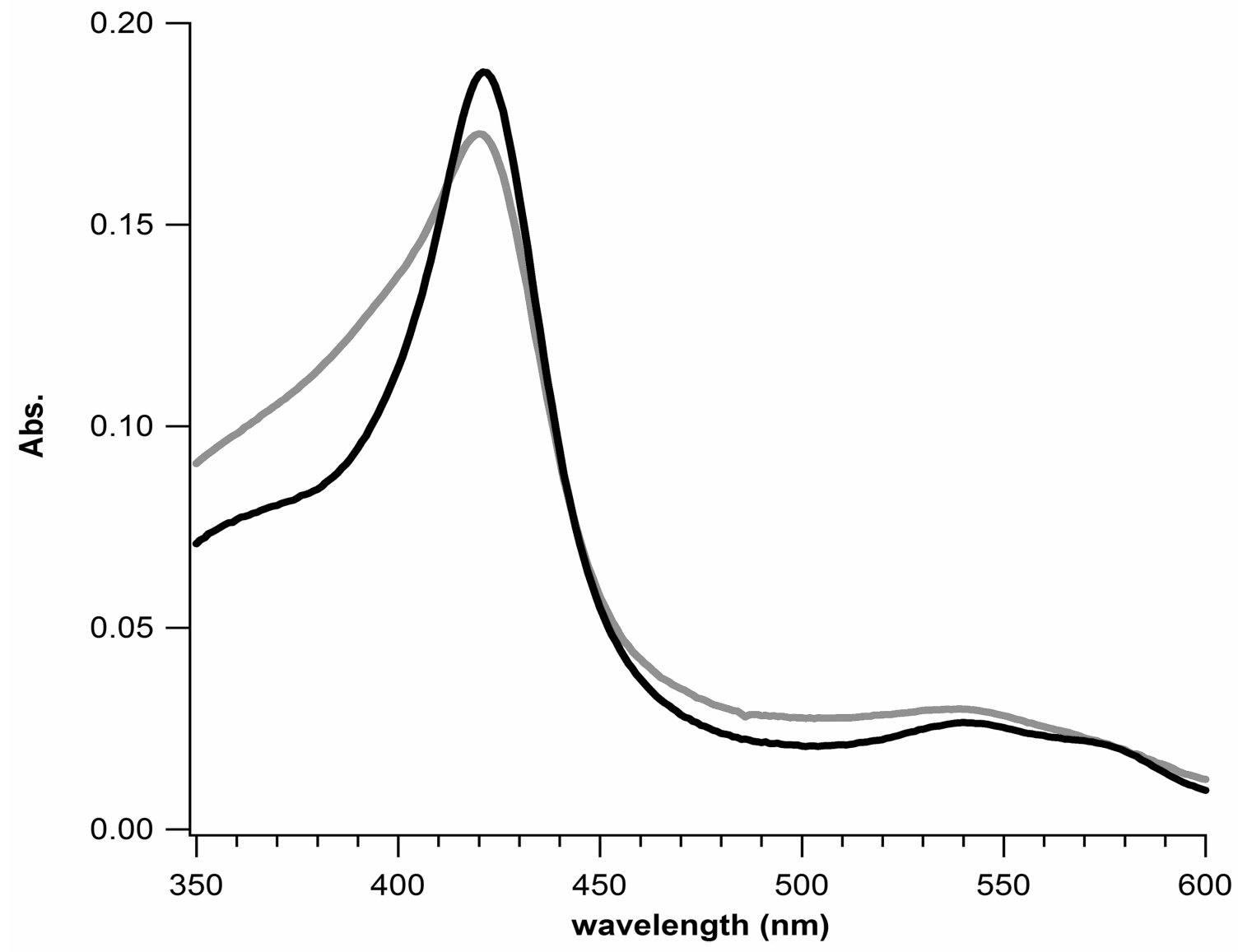

Supplemental Figure 2: Large copy of figure 4a. $2.2 \mu \mathrm{M} \Delta 114$ alone (black) and with stoichiometric 5 (gray). 


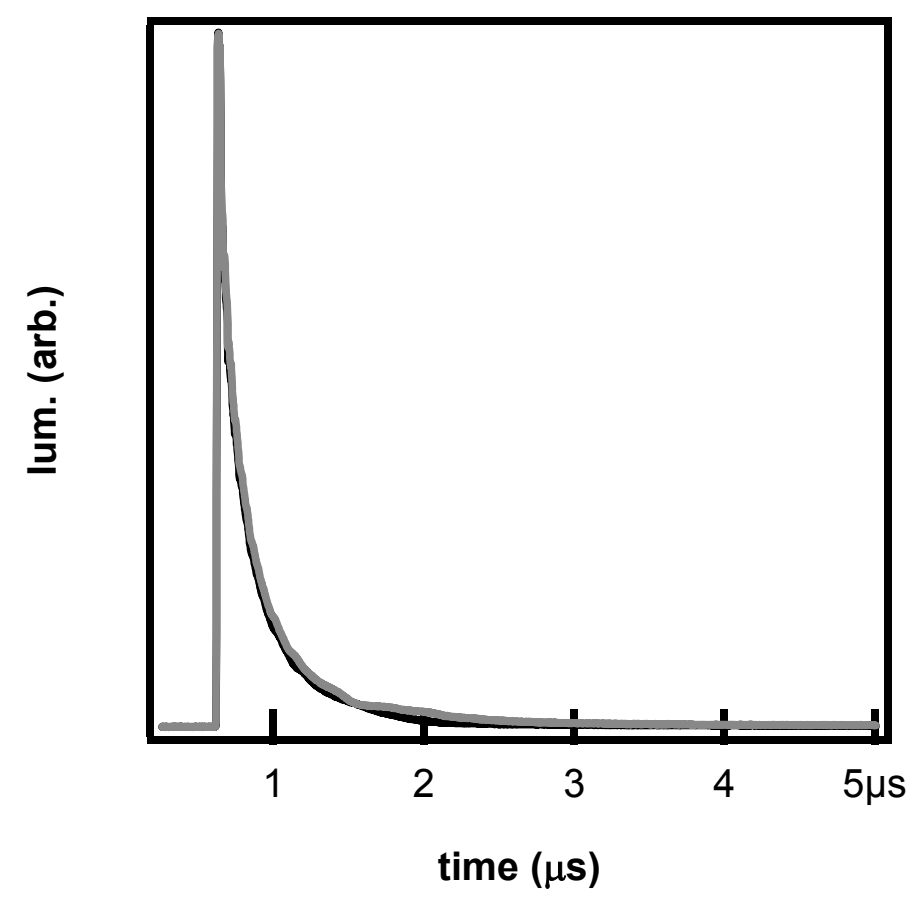

Supplemental Figure 3: Transient luminescence decay data for a 2:1 mixture of 3 with $\Delta 65$ (black) and a 2:1:1 mixture of 3,4 and $\Delta 65$ (gray). In the second mixture, 4 was shown by UV/vis to be bound at the active site. Because the luminescence decay of $\mathbf{3}$ was not disturbed by the binding of $\mathbf{4}$, these traces indicate that $\mathbf{3}$ does not bind in the active site channel. 